\title{
Supporting Information for: Direct Phase Equilibrium Simulations of NIPAM Oligomers in Water
}

\author{
Vitalie Boţan, ${ }^{\dagger}$ Vincent Ustach, ${ }^{\ddagger}$ Roland Faller, ${ }^{\dagger}$ and Kai Leonhard ${ }^{*}, \dagger$ \\ Lehrstuhl für Technische Thermodynamik, RWTH Aachen University, Schinkelstr. 8, \\ 52062 Aachen, Germany, and Department of Chemical Engineering and Materials \\ Science, University of California Davis, One Shields Ave, Davis, CA 95616, USA \\ E-mail: Kai.Leonhard@Itt.rwth-aachen.de
}

Phone: +49 (0)241 8098174. Fax: +49 (0)241 8092255

\footnotetext{
${ }^{*}$ To whom correspondence should be addressed

${ }^{\dagger}$ Lehrstuhl für Technische Thermodynamik, RWTH Aachen University, Schinkelstr. 8, 52062 Aachen,

${ }^{\ddagger}$ Department of Chemical Engineering and Materials Science,University of California Davis, One Shields Ave, Davis, CA 95616, USA
} Germany 


\section{Full Voronoi analysis}

a)

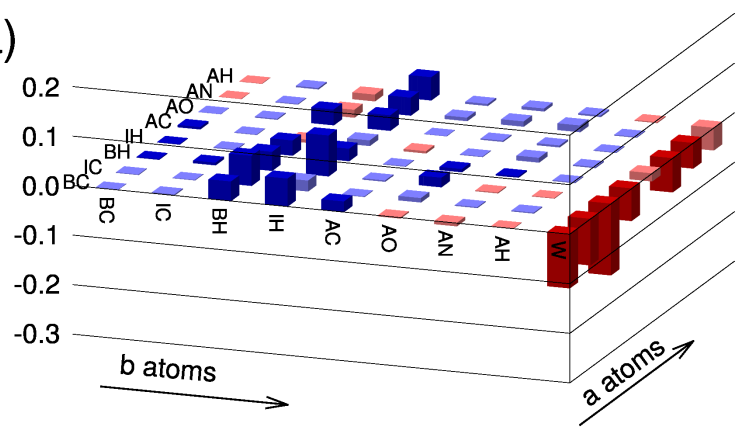

c)

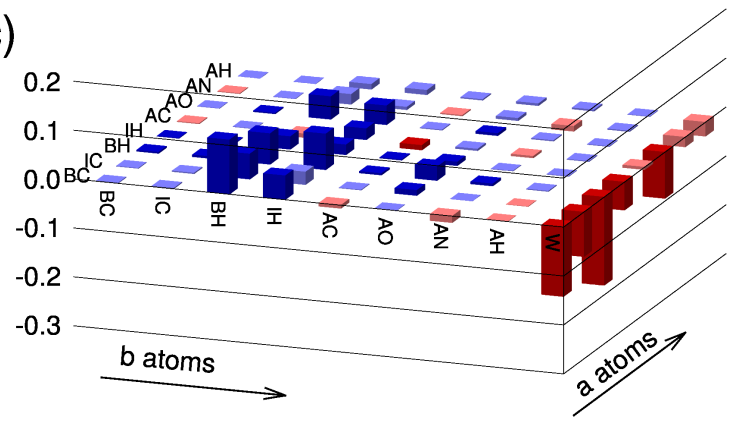

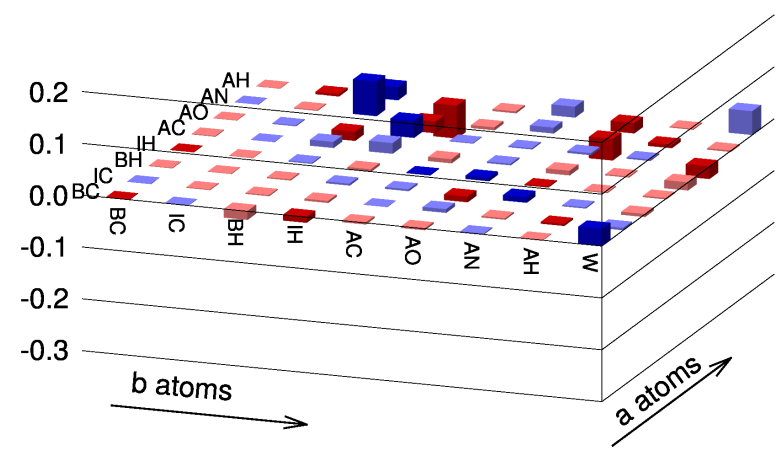

d)

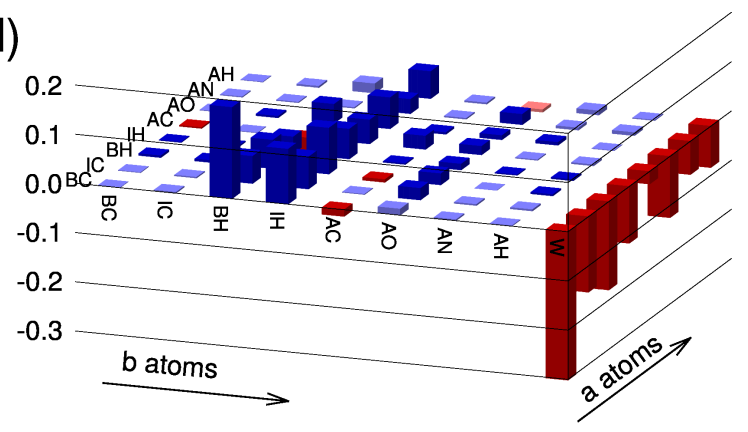

Figure S1: Top left: $\Delta\left\langle p_{a b}\right\rangle, 280 \mathrm{~K}$ compact $-280 \mathrm{~K}$ extended. Top right: $\Delta\left\langle p_{a b}\right\rangle, 290 \mathrm{~K}$ extended - $280 \mathrm{~K}$ extended. Bottom left: $\Delta\left\langle p_{a b}\right\rangle, 290 \mathrm{~K}$ compact $-280 \mathrm{~K}$ extended. Bottom right: $\Delta\left\langle p_{a b}\right\rangle, 320 \mathrm{~K}$ compact $-280 \mathrm{~K}$ extended. Each bar is the $\Delta\left\langle p_{a b}\right\rangle$ between the extended chain at $280 \mathrm{~K}$ and the other states. The rows extending into the figure represent the $a$ atoms and the columns ranging from left to right represent the $b$ atoms. Blue indicates a positive $\Delta\left\langle p_{a b}\right\rangle$. Red indicates a negative $\Delta\left\langle p_{a b}\right\rangle$. The dark bars are statistically significant changes. $\mathrm{BC}$ is backbone carbon, IC is isopropyl carbon, $\mathrm{BH}$ is backbone hydrogen, IH is isopropyl hydrogen, $\mathrm{AC}$ is amide carbon, $\mathrm{AO}$ is amide oxygen, $\mathrm{AN}$ is amide nitrogen, $\mathrm{AH}$ is amide hydrogen, and $\mathrm{W}$ is water. 


\section{Additional structural analysis}

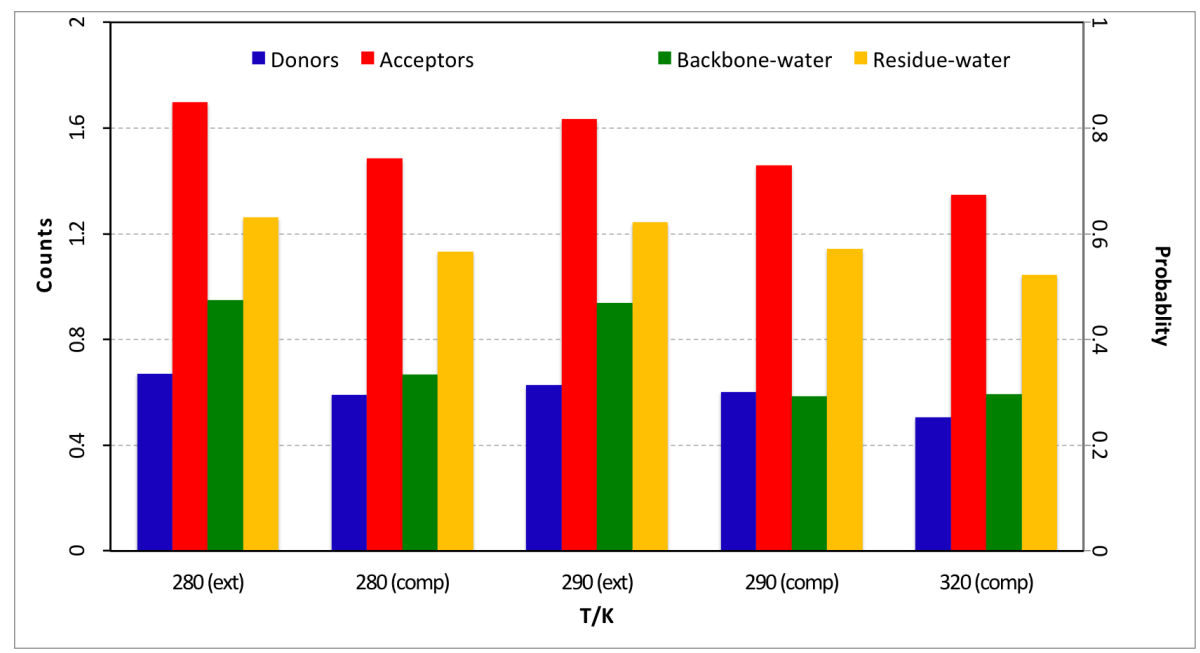

Figure S2: Average solvation numbers for a single 30mer OPLS-AA NIPAM chain in SPC/E water in different conformations at 3 different temperatures. Blue bar: average number per monomer of polymer-water hydrogen bonds with amine hydrogen as a donor. Red bar: average number per monomer of hydrogen bonds between carbonyl oxygen and water hydrogens, i.e polymer being acceptor. Green bar: average contact probability of backbone atoms $-\left(\mathrm{CH}_{2}-\mathrm{CH}\right)$ - with water atoms from BRW Voronoi analysis. Yellow bar: average contact probability of residue atoms $-\left(\mathrm{CONHC}_{3} \mathrm{H}_{7}\right)$ with water atoms for BRW Voronoi analysis. The relative error for all bars is below $2 \%$ and is not shown here. Note the different axis scales. 


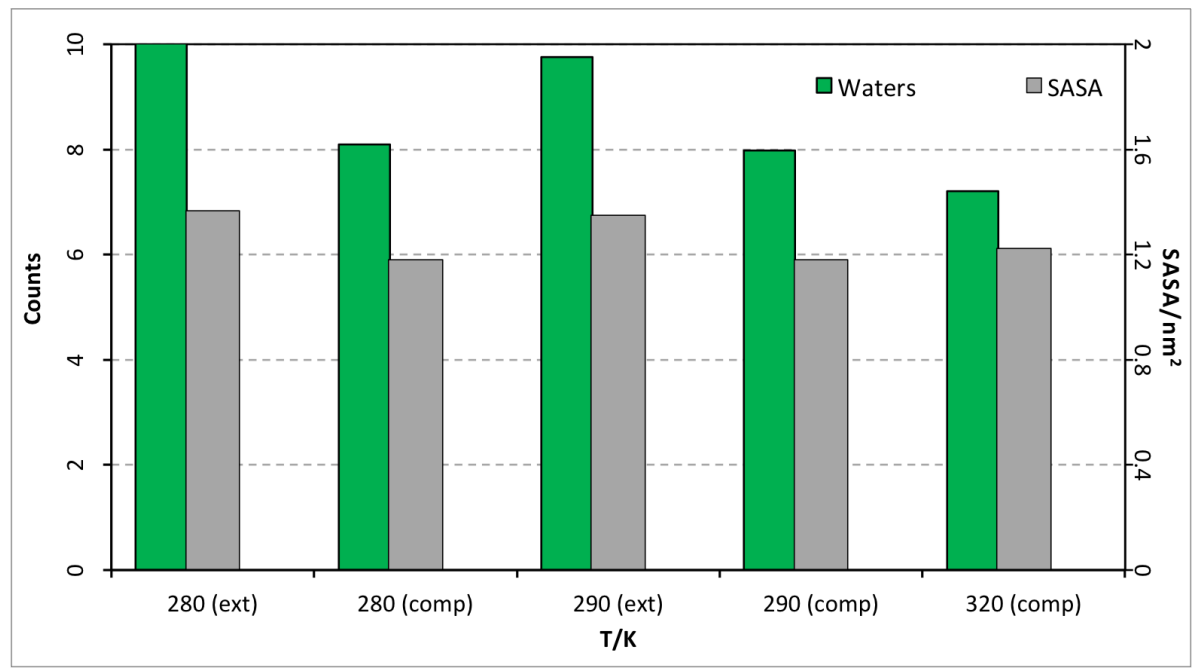

Figure S3: Green bar: average number of water molecules per monomer in the first hydration shell, defined as a distance from heavy polymer atoms to the position of the first minimum $(4.2 \AA)$ in the radial distribution function of heavy polymer atoms and water oxygen (not shown) at 3 different temperatures. Grey bar: average SASA per monomer at 3 different temperatures and conformations. Note the different scales of axis. The relative error for all bars is below $1.5 \%$ and is not shown here. 


\section{Results for additional force fields and system sizes}
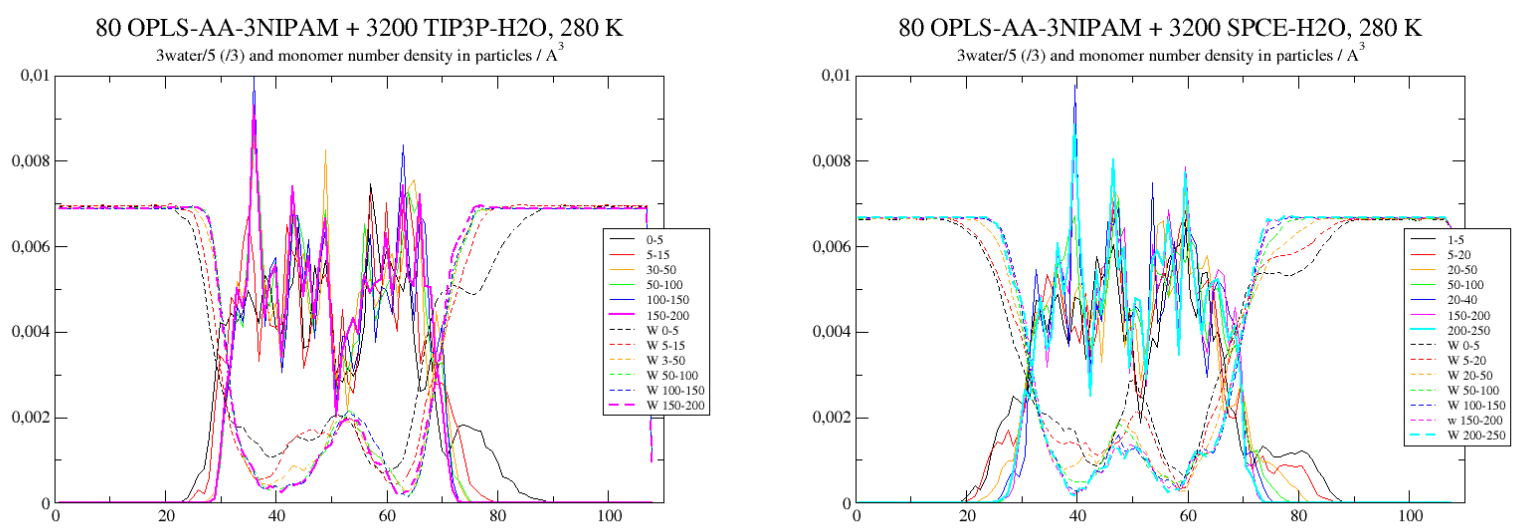

Figure S4: Density profiles of NIPAM trimers and water in water at $280 \mathrm{~K}$ started from an already inhomogeneous density distribution. Left: OPLSAA+TIP3P, right: OPLS$\mathrm{AA}+\mathrm{SPC} / \mathrm{E}$. After about 150-200 ns, the density profiles seem to become stationary. Since diffusivites are very slow in this state, and the water profile is not flat in the polymmer-rich phase, this may not be the equilibrium state but a glassy one. 

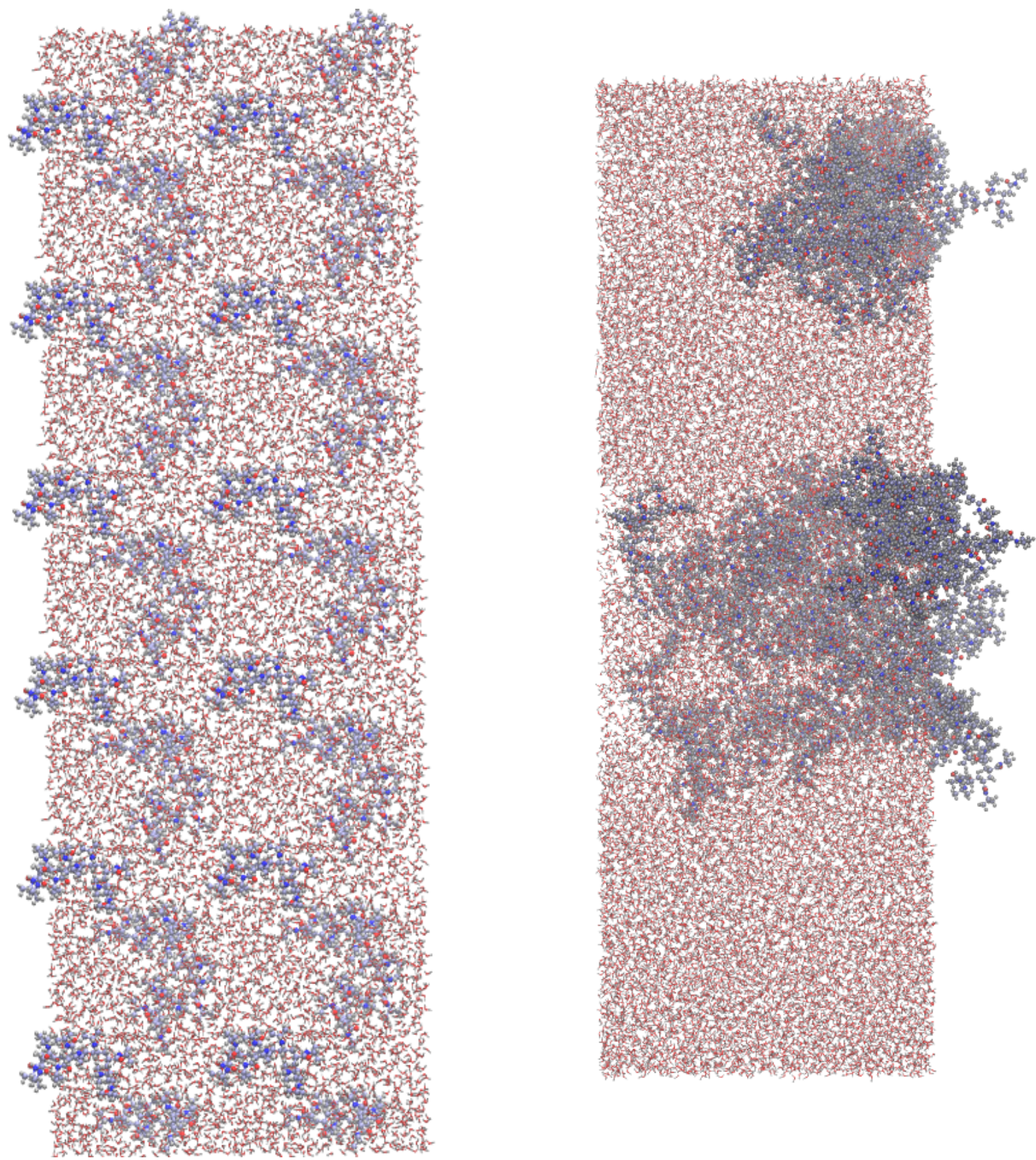

Figure S5: Results of the simulations of 72 OPLS-AA NIPAM decamers in 25584 SPC/E water molecules. Left: The simulations started with a homogeneous density distribution of decamers and water. Right: Within the accessible time (200 ns), a clear separation into two large clusters can be observed but not the formation of a single polymer-rich phase. Hydrogen atoms are depicted in grey, oxygen ones in red, carbon ones in steel, blue and nitrogen ones in blue. 


\section{Simulation details}

Detailed geometry and potential parameter information can be found in Table S1.

Table S1: List of files included in the supporting information.

\begin{tabular}{ll}
\hline \hline filename & description \\
\hline 30mer-OPLS-AA-parameters.txt & 30mer geometry and OPLS-AA parameters in LAMMPS format \\
decamer-OPLS-AA-parameters.txt & decamer geometry and OPLS-AA parameters in LAMMPS format \\
trimer-OPLS-AA-parameters.txt & trimer geometry and OPLS-AA parameters in LAMMPS format \\
trimer-amber94-parameters.txt & trimer geometry and amber94 parameters in LAMMPS format \\
water-spce-parameters.txt & water geometry and SPC/E parameters in LAMMPS format \\
water-TIP3P-parameters.txt & water geometry and TIP3P parameters in LAMMPS format \\
amber94+TIP3P_production.Imp & LAMMPS input file for a production run (after thermalization) \\
OPLS-AA+SPCE_production.lmp & LAMMPS input file for a production run (after thermalization) \\
dimer_topol.top & Dimer geometry and G45A3 parameters in Gromacs format \\
dimer_production.mdp & Gromacs input file for a production run (after thermalization) \\
\hline \hline
\end{tabular}

